\title{
Purpura Fulminans: Meningococcemia Manifestation
}

\author{
Walter Chaves S, ${ }^{1}$ Alejandro Dueñas, ${ }^{2 *}$ Carolina Rodríguez E, ${ }^{3}$ Edwin Núñez, ${ }^{4}$ Nelson Amador ${ }^{5}$ \\ ${ }^{1}$ Director Internal Medicine program, University Foundation of Health Sciences, San Jose Hospital, Colombia \\ ${ }^{2}$ Resident of Internal Medicine, University Foundation of Health Sciences, San Jose Hospital, Colombia \\ ${ }^{3}$ Physician Mederi Hospital, Colombia \\ ${ }^{4}$ Physician Los Nogales Clinic, Colombia \\ ${ }^{5}$ Physician Los Nogales Clinic. Colombia
}

\begin{abstract}
Background: Neisseria meningitidis is the most frequent etiologic agent of meningococcemia and meningitis. In both scenarios it is capable to produce clinical manifestations of great importance. The purpura fulminans is part of the cutaneous signs caused by this bacterial agent. It begins as a cutaneous hemorrhage and necrosis due to vascular thrombosis and disseminated intravascular coagulation secondary to immunological, genetic, and vascular events. Being in this infectious disease a factor of poor prognosis.

Case presentation: We present a case of purpura fulminans where the etiological agent isolated by blood culture was Neisseria meningitidis in a Patient at the Hospital de San José in the city of Bogotá. The patient shows on physical examination erythematous macula type lesions with irregular edges like purpuric lesions. The isolated bacteria was Neisseria meningitidis and the diagnosis of meningococcemia was did.

Conclusion: Meningococcemia it is considered that the most frequent etiologic agent of this entity is Neisseria meningitidis. Typically, it can start as a cutaneous hemorrhage and necrosis due to vascular thrombosis and disseminated intravascular coagulation, being a presentation a factor of poor prognosis.
\end{abstract}

Keywords: Purpura fulminans, Meningococcus, Meningococcemia, Meningitis

Abbreviations: PF: Purpura Fulminans; CRP: C reactive protein; DIC Disseminated intrvascular coagulation

\section{Background}

Meningococcal infections are secondary to colonization in the upper respiratory tract, consequently reaching the bloodstream to constitute a meningococcemia: presenting a broad spectrum of clinical manifestations, like cases of purpura fulminans. About meningococcemia it is considered that the most frequent etiologic agent of this entity is Neisseria meningitidis. Typically, it can start as a cutaneous hemorrhage and necrosis due to vascular throm- bosis and disseminated intravascular coagulation, being a presentation a factor of poor prognosis. In the following case report will present the case of a male patient, who has purple fulminans as a cause of meningococcemia.

\section{Case Presentation}

A 30-year-old male patient presented with 5 day of odynophagia, asthenia, rhinorrhea, non-emetizing non- caino dry cough without the presence of febrile peaks or any other symptoms. Two days after presents, intermittent febrile peaks of nocturnal predominance, with maximum peak up to $39.7^{\circ}$, associated with nau-

\begin{tabular}{|l|l|}
\hline \hline Quick Response Code: & *Corresponding author: Alejandro Duenas, University Foundation of Health Sciences, San \\
Jose Hospital, Colombia \\
Received: 20 September, 2021 \\
Citation: Walter Chaves S, Dueñas A, Carolina Rodríguez E, Núñez E, Amador N. Purpura \\
Fulminans: Meningococcemia Manifestation. SOJ Med Clin Case Rep. 2021;1(1):1-4. DOI: \\
10.53902/SOJMCCR.2021.01.000506
\end{tabular}


sea, vomiting, diarrhea without mucus or blood. For this reason, the patient visited physician: who indicate symptomatic management with acetaminophen and butyl bromide of hyoscine, considering possible viral infection. Subsequently, he refers an increase on symptoms and the appearance of erythematous macula type lesions with irregular edges no necrotic center and approximately size of $40 \mathrm{~mm}$ by $35 \mathrm{~mm}$, predominantly in the extremities, without compromising mucous membranes. Additionally, referred oppressive left hemicranean headache with occipital irradiation of intensity 7/10 that exacerbates with cough; also, joint pain predominantly in small joints in hands and feet. As a significant epidemiological link, a month ago he traveled to the Caribbean region (Santa Marta) where he stayed for 3 days.

On the examination he was with tachycardia (102pm), tachypneic (22rpm), febrile $/\left(38.3^{\circ}\right)$, bilateral conjunctival injection, congestive erythematous oropharynx, no cardiopulmonary cramps and we found in the skin lesions like purpuric confluent macules, that do not disappear at digit pression, no compromise of the face, or mucous membranes, predominantly in the lower and upper limbs, neurological examination without motor or sensory deficit, without evidence of meningeal signs.
Laboratories initially requested, showed a full blood count with leukocytosis $(21,200)$, Neutrophilia $(18,500)$ rod neutrophils (21\%), CRP (50), with prolongation of TP 22.5 over control of 14, preserved renal function, normal uranalysis. With the history of headache, arthralgia, odynophagia, purpura and the physical examination findings tachycardia, fever, tachypnea, purpuric lesions, added to the blood laboratories findings: meningococcemia was considered. Therefore, we treated empirically with broad spectrum antibiotic (Ceftriaxone), we took bloodstream cultures and request lumbar puncture.

The findings in cerebrospinal fluid showed: transparent liquid, 1Xmm3 leukocytes, red blood cells 0 , negative $\mathrm{KOH}$, non-reactive serology, glucose 56/serum 60. GLUCOSE INDEX 0.9, proteins 50. With these findings Meningoencephalitis was ruled out.

After 24 hours of treatment we recieve bloodstream cultures: Gram negative diplococci growth is reported in the 2 aerobic blood cultures, the isolated germ was Neisseria meningitidis.

Alongside this, his symptoms were better we continue antibiotical treatment, there were no side effects from this. Finally he recovered and was subsequently leading hospital discharge (Figure 1).

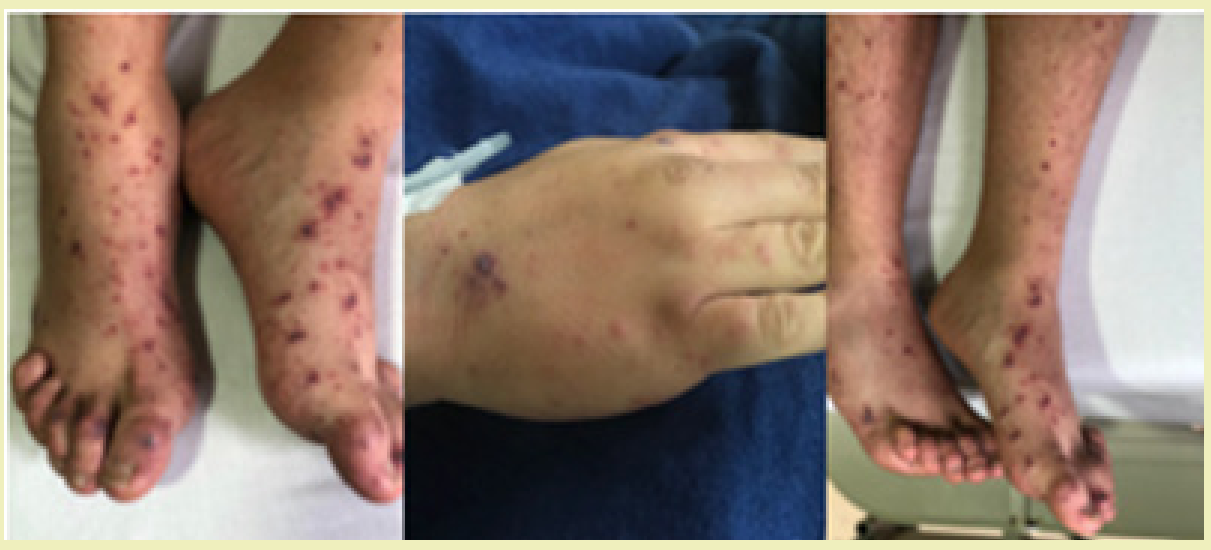

Figure 1: Purpuric lesions on feet, right hand.

\section{Discussion}

Meningococcal infections can be endemic and epidemic, being highly frequent, there are two clinical manifestations of the disease: meningitis and meningococcemia. To cause the disease, the pathogen colonizes the nasopharynx, advance into the bloodstream and survives to the host defense mechanisms in the intravascular space, crosses the blood-brain barrier and replicates in the subarachnoid space. It is very important to know the host inflammatory factors that may contribute to the pathophysiological cascade, as this helps establish a more specific treatment. ${ }^{1}$

Meningitis is an inflammatory disease of the meninges, tissues around the brain and spinal cord and is defined by an abnormal number of leukocytes counts in the cerebrospinal fluid (CSF). The meninges consist of three parts: pia mater, arachnoid and dura mater. Bacterial meningitis reflects infection of the arachnoid, CSF in the subarachnoid space and the cerebral ventricles. ${ }^{1}$ This disease has a wide range of clinical manifestations; however, we will talk about skin manifestations that are observed in cases of meningococcemia.

\section{Clinical skin manifestations}

Some bacteria, particularly Neisseria meningitidis, can cause characteristic skin manifestations such as palpable petechiae and purpura. A petechial rash is not specific for meningococcal infection and some patients with meningitis have a maculopapular rash. ${ }^{1}$ 


\section{Purpura fulminans}

It is a severe complication of meningococcal disease, occurring in approximately $15-25 \%$ of those due to the development of endotoxins; being this manifestation of bad prognosis and meaning a high risk of mortality.

Neisseria meningitidis is until now the main cause of infectious $\mathrm{PF}$, followed by infection by other encapsulated bacteria such as Streptococcus pneumoniae and Haemophilus Influenzae. During severe meningococcemia other organs may be involved such as the kidneys, heart and adrenal glands (causing Waterhouse-Friederichsen syndrome). On the other hand, if patients do not suffer from $\mathrm{PF}, 42 \%$ to $70 \%$ of patients suffering from meningococcemia have a skin purpuric rash, the initial lesion of $\mathrm{PF}$, highlighting the prothrombotic nature of meningococcal invasive infections. ${ }^{2,3}$

PF is a potentially lethal disorder that in the acute onset produces cutaneous bleeding and necrosis secondary to vascular thrombosis and disseminated intravascular coagulation. These manifestations are characterized by skin pain with subsequent erythema and petechiae. When the ecchymoses develop evolve into indurated, painful, well-marked purple papules with erythematous borders, these areas progress to necrosis with formation of bullas and vesicles: Gangrenous necrosis can continue with extension to the subcutaneous tissue and occasionally involves muscle and bone.

During meningococcemia the levels of circulating bacteria, endotoxin and proinflammatory cytokines are very high and correlate with the severity of the disease and alterations in coagulation. In addition, the meningococcal liposaccharide is usually composed by a lipid A which is optimal for the recognition of TLR4 receptors and may be associated with the meningococcal prothrombotic state.,4

The adhesion of meningococcus to endothelial cells is a key event for thrombosis of the skin microvasculature, explaining that the CID is not the only contributing factor for PF. Extensive endothelial colonization is specific to Neisseria meningitidis, this may explain why purpura and PF are more frequent during meningococcemia than in other infections. Adhesion can intensify the prothrombotic endothelial response such as overexpression of the membranous tissue factor, the exposure of negative phospholipid charges by activating the contact pathway, the release of von Willebrand factor or the release of procoagulant circulating microparticles. Meningococcal adhesion may also alter the antithrombotic or profibrinolytic endothelial responses by: downregulation of thrombomodulin membrane expression, endothelial C protein receptor, preventing the release of tissue plasminogen activator, promoting the release of PAI-1 (plasminogen activator 1 inhibitor). ${ }^{3}$

In the other hand its known that PF may be the consequence of a homozygous congenital defect in the coagulation system, in pro- tein $\mathrm{C}$ and $\mathrm{S}$, usually observed as a post-infectious consequence of an autoimmune disorder and may be associated with severe infection. $^{3-5}$

Protein C dysfunction is critical for the appearance of PF. Protein $\mathrm{C}$ is vitamin $\mathrm{K}$ dependent and circulates in the plasma as an inactivated zymogen. Once activated, protein $\mathrm{C}$ requires protein $\mathrm{S}$ as a cofactor, for its anticoagulant functions. ${ }^{6,7}$

\section{Diagnosis}

The basis of the diagnosis is fundamentally clinical, based on symptoms and signs of the patient. Between the differential diagnosis includes warfarin-induced cutaneous necrosis, cryoglobulinemic vasculitis, catastrophic antiphospholipid syndrome, heparin-induced thrombocytopenia and Henoch-Schonlein purpura. Additionally, patients with FP have findings in the laboratories such, prolonged coagulation times, decreased fibrinogen, elevated D-dimer and thrombocytopenia. These patients also have elevated C-reactive protein (CRP) in the context of low globular sedimentation rate (ESR). This PCR-VSG dissociation is due to relative or absolute hypofibrinogenemia in the context of DIC, which leads to an artificially decreased ESR. In addition, these patients have decreased levels of protein C and S. Levels of protein C below $40 \%$ are suspected of $\mathrm{PF}^{4}$

The knowledge of this pathology gives a light for the therapy in meningococcemia that should be directed to design specific therapies to prevent or limit vascular thrombosis together with antibiotics and intensive care unit. ${ }^{3}$

\section{Treatment}

As previously mentioned, PF is caused as a complication of meningococcal disease and it's the reason why it is very important to start early and appropriate treatment to improve the prognosis of meningococcal infections. Antibiotic management should be initiated as soon as possible because in meningococcal sepsis there are extremely high bacterial loads and the delay in the onset may result in higher rates of complications and mortality. ${ }^{8-10}$ Blood cultures should be performed before the start treatment with the goal to guide appropriately the treatment.

The treatment consists of administering third generation cephalosporins such as cefotaxime or ceftriaxone. ${ }^{8}$ The third generation cephalosporins are the treatment, the recommended dose is ceftriaxone $2 \mathrm{~g}$ IV every 12 hours or cefotaxime $2 \mathrm{~g}$ IV every 4 to 6 hours for seven days. ${ }^{9}$ Another option for meningococcal meningitis is penicillin $\mathrm{G}$ if the isolated germ shows sensitivity to it. It can be used if it has a MIC of $<0.1 \mathrm{mcg} / \mathrm{ml}$. For isolates with MIC $>0.1$ to $1.0 \mathrm{mcg} / \mathrm{ml}$, penicillin treatment is effective, but third generation cephalosporins are preferred. It can be administered IV or IM. The usual dose is 300,000 IU/kg per day, with an upper limit of 24 mil- 
lion units per day. The most usual dose is 4 million IU IV every four hours. ${ }^{8}$

\section{Conflict of Interests}

None.

\section{Funding}

None.

\section{Consent for Publication}

Informed consent was obtained from the parents of the patient to publish this case in a medical journal.

\section{Ethical Approval}

Ethical approval is not required at our institution for publishing a case report in a medical journal.

\section{References}

1. Scheld WM, Koedel U, Nathan B, et al. Pathophysiology of bacterial meningitis: mechanism(s) of neuronal injury. I Infect Dis. 2002;186(2):S225-S233.
2. Darmstadt G. Acute infectious purpura fulminans: pathogenesis and medical management. Pediatric Dermatology. 1998;15(3):169-183.

3. Lécuyer H, Borgel D, Nassif X, et al. Pathogenesis of meningococcal purpura fulminans. Pathog Dis. 2017;75(3).

4. Colling ME, Bendapudi PK. Purpura Fulminans: Mechanism and Management of Dysregulated Hemostasis. Transfus Med Rev. 2018;32(2):69-76.

5. Smith OP, White B. Infectious purpura fulminans: diagnosis and treatment. British Journal of Haematology, 1999;104:202-207.

6. Faust SN, Levin M, Harrison OB, et al. Dysfunction of endothelial protein $\mathrm{C}$ activation in severe meningococcal sepsis. $N$ Engl J Med. 2001;345(6):408-416.

7. Chenaille PJ, Horowitz ME. Purpura Fulminans. Clinical Pediatrics. 1989;28(2):95-98.

8. Tunkel AR, Hartman BJ, Kaplan SL, et al. Practice guidelines for the management of bacterial meningitis. Clin Infect Dis. 2004;39(9):12671284.

9. Marhoum El Filali K, Noun M Chakib A. et al. Ceftriaxone versus penicillin $\mathrm{G}$ in the short-term treatment of meningococcal meningitis in adults. Eur J Clin Microbiol Infect Dis. 1993;12:766. 